\title{
Comparative Abundance of the Large Subunit of Rubisco in Aerial Organs of the Japanese Morning Glory [Ipomoea (Pharbitis) nil] Suggests a Photosynthetic Role for Sepals
}

\author{
Carole L. Bassett \\ U.S. Department of Agriculture, Agricultural Research Service, Appalachian Fruit Research Station, \\ Kearneysville, WV 25430
}

\begin{abstract}
AdDITIONAL INDEX WORDS. carbon assimilation, immunoblotting, differential expression
AbSTRact. Levels of the large subunit (LSU) of rubisco (ribulose 1,5 bisphosphate carboxylase/oxygenase) were measured in vegetative and floral organs of 'Violet' Japanese morning glory (Ipomoea [Pharbitis] nil Roth). Identification of the LSU in polypeptides separated by two-dimensional gel electrophoresis allowed estimation of the relative abundance of this polypeptide in the organs examined. Further quantitation was achieved by immunoblotting protein extracts either alone or in combination with various amounts of extracts from other organs. The amount of LSU decreased in the order leaves $>$ cotyledons $>$ sepals $>$ corollas > androecium, gynoecium. The relative abundance of LSU in sepals (74\%) compared to photosynthetically competent organs [leaves $(\mathbf{1 0 0 \%})$ and cotyledons $(\mathbf{8 1 \%})$ ] suggests that sepals may be photosynthetically competent in supporting development of the other floral organs.
\end{abstract}

In higher plants carbon is partitioned from organs that fix $\mathrm{CO}_{2}$ or store long-term carbohydrate reserves (source organs) to those organs or plant parts that require imported carbon for growth and maintenance (sink organs). Virtually all organs act as sinks at some time or another during their development. For example, roots and young leaves of tomato were shown to compete more effectively for assimilates than flowers; however, when tomatoes began to fruit, the fruit were stronger sinks than either roots or young leaves (Bravdo et al., 1977; Ho, 1984). The competitiveness of a particular organ or tissue as a sink is related to the number of cells in the organ and its physiological state, and is therefore a consequence of both genetic determination and environmental influences (Ho, 1988).

Although flowers and fruit are usually regarded as sink organs, some are theoretically capable of limited photosynthesis from early development up to the onset of maturity. For example, the chlorophyll content of petunia (Petunia hybrida L.) corollas increased during development to a maximum just before anthesis, and chloroplasts isolated from corollas after anthesis were capable of carrying out light-induced electron transport, although at onethird the rate of green leaves (Weiss et al., 1989). In another study chloroplasts from red-pigmented corollas, bracts and leaves of several different plants demonstrated Hill reaction activity that was as high or higher on a per chlorophyll basis than chloroplasts from green leaves (Sharma, 1980). Similarly, studies of carbon assimilation in fruit and flowers indicate that these organs can refix respired $\mathrm{CO}_{2}$ (Bazazz et al., 1979; Hedley et al., 1975; Pavel and DeJong, 1993; Quebedeaux and Chollet, 1975; Vu et al., 1985; reviewed in Blanke and Lenz, 1989) and in at least one case contribute to net photosynthesis (Flinn et al., 1977).

Received for publication 27 Apr. 1998. Accepted for publication 8 Sept. 1998. The gifts of antisera to the large subunit of rubisco (J.R.Y. Rawson, British Petroleum) and purified corn rubisco (Bonnie J. Reger, USDA-ARS) are gratefully acknowledged. I am indebted to Cheryle Mothershed for her expert technical assistance. Use of a trade name or trade mark does not imply endorsement to the exclusion of other, similar products. This paper is dedicated to the memory of Bonnie Reger, colleague and friend. The cost of publishing this paper was defrayed in part by the payment of page charges. Under postal regulations, this paper therefore must be hereby marked advertisement solely to indicate this fact.
Floral organs are considered to be modified leaves based on their developmental origin and morphology. In 'Violet' Japanese morning glory, pairwise comparisons of polypeptide profiles from aerial organs have indicated that sepals more closely resemble leaves than other floral organs (Bassett et al., 1988), raising a question as to whether sepals can function like leaves by carrying out limited photosynthesis. Several factors, including ribulose 1,5 bisphosphate levels, and the activation status and concentration of rubisco can limit photosynthesis (reviewed in Woodrow and Berry, 1988). Therefore, the observed low abundance of this enzyme in nonleaf tissues may be a key factor limiting their photosynthetic capability. To explore this question further, the relative abundance of the large subunit (LSU) of rubisco, EC4.1.1.39, the catalytically active subunit of the primary $\mathrm{CO}_{2}$ fixation enzyme in plants, was determined qualitatively and quantitatively in various aerial organs to estimate the functional limit contribution of each organ to carbon fixation.

\section{Materials and Methods}

Growth AND COllection of Plant material. Plants were grown in growth chambers under the conditions described by Bassett et al. (1988). Briefly, sulfuric acid-treated seeds were germinated overnight and subsequently planted in artificial soil (day 1). Seedlings were grown under continuous light $\left(190 \mu \mathrm{mol} \cdot \mathrm{m}^{-2} \cdot \mathrm{s}^{-1} \mathrm{PAR}\right)$ at $25^{\circ} \mathrm{C}$. Flowers were induced by subjecting 4 -d-old plants to a single short-day ( $16 \mathrm{~h}$ dark). After the dark inductive period, plants were maintained under long days ( $16 \mathrm{~h}$ light $/ 8 \mathrm{~h}$ dark). Tissues were collected from 10 plants, pooled and immediately frozen in liquid nitrogen for storage at $-80^{\circ} \mathrm{C}$. Ten fully expanded, mature leaves, 20 cotyledons and 50 flowers were sampled 29 to $31 \mathrm{~d}$ after planting. Whorls of floral organs were dissected from flowers and pooled. Stems were taken from 29-d-old plants in regions where purple pigmentation was fully developed (mature stems). In 'Violet', cotyledons persist until $\approx 50 \mathrm{~d}$ from planting at which time they begin to show signs of senescence.

ISOLATION AND CHARACTERIZATION OF PROTEINS. SDS-extractable proteins were prepared from each tissue as previously described (Bassett et al., 1991) keeping the ratio of fresh weight:volume 
extraction buffer the same for each tissue. Polypeptides were separated by nonequilibrium $\mathrm{pH}$ gradient electrophoresis [NEPHGE] using standard protocol for the first dimension (O'Farrell, 1975) with modifications previously described (Bassett et al., 1988). Gels were run for $3200 \mathrm{~V} \cdot \mathrm{h}^{-1}$ in the first dimension generating a linear gradient from $\mathrm{pH} 4$ to 10 . Polypeptides were separated in the second dimension by SDS-polyacrylamide gel electrophoresis [SDS-PAGE] (Laemmli, 1980) through a 12.5\% polyacrylamide slab gel. Polypeptides were visualized by silver staining according to Oakley et al. (1980). The LSU of rubisco was identified by its distinctive pI (Johal and Chollet, 1983), size (Gray and Kekwick, 1974), and comigration with purified maize (Zea mays L.) LSU (a gift from Bonnie J. Reger, USDA-ARS).

IMmunoblotTING OF POLYPEPTIDEs. Polypeptides separated in one dimension by SDS-PAGE were transferred to nitrocellulose filters using a vertical TransBlot cell (LKB, Switzerland) under conditions described by Cameron and Bassett (1988). Rabbit antiserum against the LSU of rubisco from Euglena sp. (a gift of J.R.Y. Rawson, British Petroleum) was diluted 1:1000 with phosphate-buffered saline (10 $\mathrm{mm} \mathrm{Na}_{3} \mathrm{PO}_{4}$ and $\left.140 \mathrm{~mm} \mathrm{NaCl}\right)$ and bound to polypeptides transferred to nitrocellulose filters previously blocked with $1 \%$ bovine serum albumin. Primary antibodies were reacted with goat antirabbit alkaline phosphatase conjugate (Sigma Chemical Co., St., Louis, Mo.), and visualized by staining for alkaline phosphatase activity (Blake et al., 1984). The antibody was checked for specificity by comparison to a preimmune serum control and for cross-reactivity against purified rubisco from maize. Patterns of LSU expression were corroborated using antichalcone synthase (anti-CHS) to examine expression of CHS on blots of the same tissues reported here (manuscript in press). Since CHS is expressed predominantly in floral reproductive organs, its expression in organs where LSU levels were low confirmed the integrity of the protein preparations and methodologies. To quantitate the LSU, band widths from the stained filters were measured under a dissecting microscope with an attached micrometer. As a check on quantitative transfer of LSU, extracts from various organs were mixed in different proportions, separated in gels and transferred to filters. Band widths of the LSU from the organ mixtures were additive as expected (data not shown).

\section{Results and Discussion}

Photosynthesis is the sum total of two highly integrated processes, i.e., light-activated electron transport and $\mathrm{CO}_{2}$ assimilation. In photosynthetically competent leaves the rate of $\mathrm{CO}_{2}$ assimilation is limited by the supply of ribulose 1,5 bisphosphate under conditions of low irradiance and high $\mathrm{CO}_{2}$ partial pressure (Laisk and Oya, 1974). However, under high light intensities and ambient or lower $\mathrm{CO}_{2}$ partial pressures, rubisco is the primary limitation to photosynthesis (von Cammerer and Edmondson, 1986). Therefore, regulation of photosynthesis in saturating light depends on the amount and activation status of rubisco.

Although leaves are the primary sites of photosynthesis, other organs such as flowers (Bazazz et al., 1979; Vu et al., 1985) and young fruit (Hedley et al., 1975; Pavel and DeJong, 1993; Quebedeaux and Chollet, 1975; reviewed by Blanke and Lenz, 1989) can carry out this process. However, in most cases the $\mathrm{CO}_{2}$ exchange data reflect refixation of respiratory $\mathrm{CO}_{2}$, rather than net photosynthesis (Hedley et al., 1975; Quebedeaux and Chollet, 1975). One exception is the reported net photosynthesis of young pea pods, where measurements indicated a rate of photosynthesis of $10 \%$ that of mature leaves (Flinn et al., 1977).

Studies of photosynthesis conducted on flowers have demon- strated the ability of corollas to carry out aspects of the light reactions (Sharma, 1980; Weiss et al. 1989). However, few studies have demonstrated $\mathrm{CO}_{2}$ assimilation by flowers or specific floral parts. In one such study, Vu et al. (1985) measured the activity of rubisco in extracts of 'Valencia' orange flowers at three developmental stages and compared these activities to leaves. In all stages tested the activity of rubisco in flowers was $\approx 10 \%$ or less than that of mature leaves. Despite the relatively low activity of rubisco in these flowers, analysis of ${ }^{14} \mathrm{C}$-labeled photosynthetic products indicated a high $(\approx 50 \%$ of compounds examined) incorporation into neutral sugars in the light. However, since the ratio of phosphoenolpyruvate carboxylase (PEPCase) activity to rubisco for all flower stages was significantly higher than in leaves, these results taken together, like those of developing fruit, probably reflect refixation of respired $\mathrm{CO}_{2}$ rather than net incorporation.

In the examples cited above, the ability to fix carbon is primarily limited by low levels and/or activity of rubisco, since activities in developing flowers, seed pods and fruit ranged from only $3 \%$ to $10 \%$ those of mature leaves (Hedley et al., 1975; Quebedeaux and Chollet, 1975; Vu et al., 1985). Similarly, examination of SDSextractable proteins from different organs of morning glory 'Violet' separated by NEPHGE indicated that the LSU was least abundant in corollas, androecia and gynoecia (Fig. 1, arrows). In contrast, stems and sepals appeared to have considerable levels of rubisco. When protein extracts from developing cotyledons were

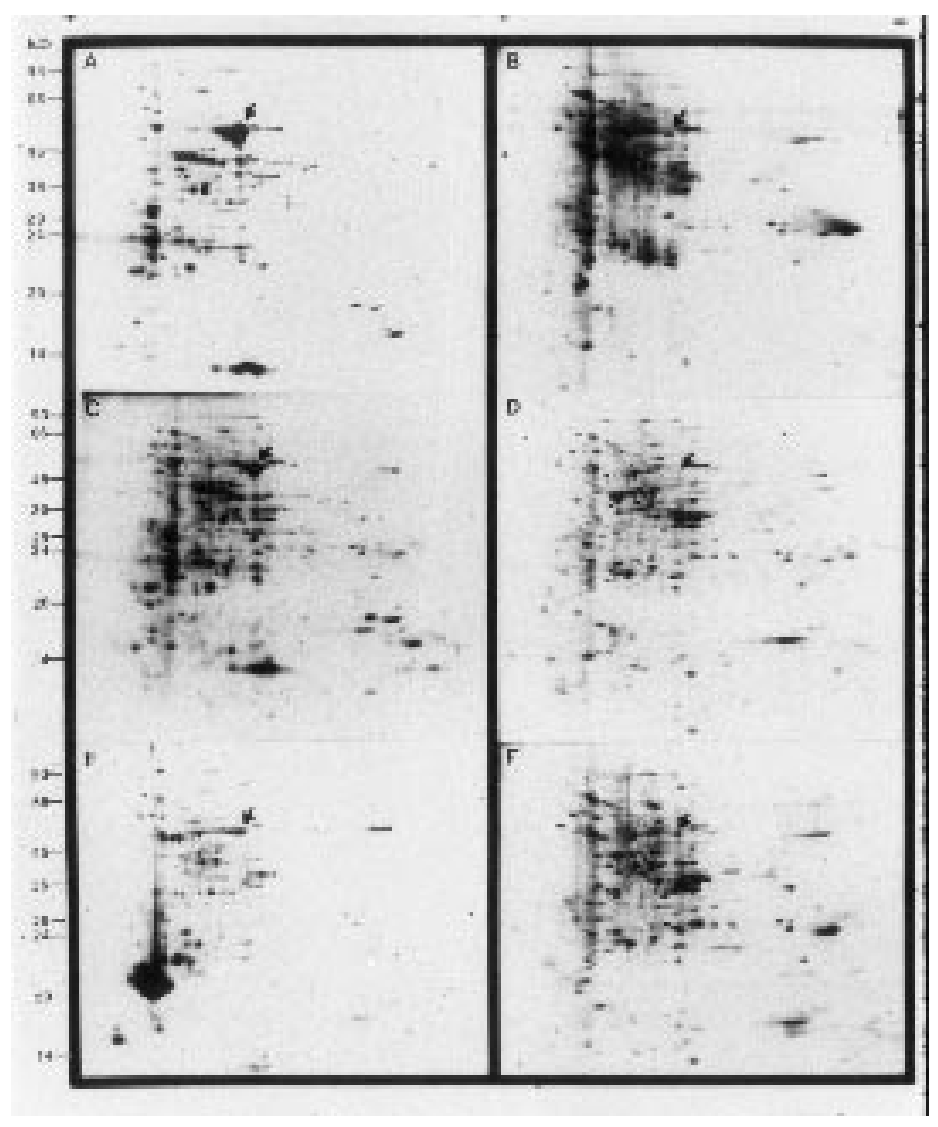

Fig. 1. Nonequilibrium pH gel electrophoresis of SDS-extracted proteins from various organs of morning glory. One hundred micrograms of protein were loaded onto each $\mathrm{pH}$ gradient gel and electrophoresed for $3200 \mathrm{~V} \cdot \mathrm{h}^{-1}$ in the first dimension. The proteins were electrophoresed in the second dimension in SDSpolyacrylamide slab gels and stained with silver nitrate for visualization. Proteins from (A) mature leaves, (B) corollas at anthesis, (C) sepals at anthesis, (D) androecium at anthesis, (E) mature stems, and (F) gynoecium at anthesis. Molecular size markers are indicated to the left of each gel set. Anode (+), cathode $(-)$. Arrows indicate the location of the large subunit of rubisco. 


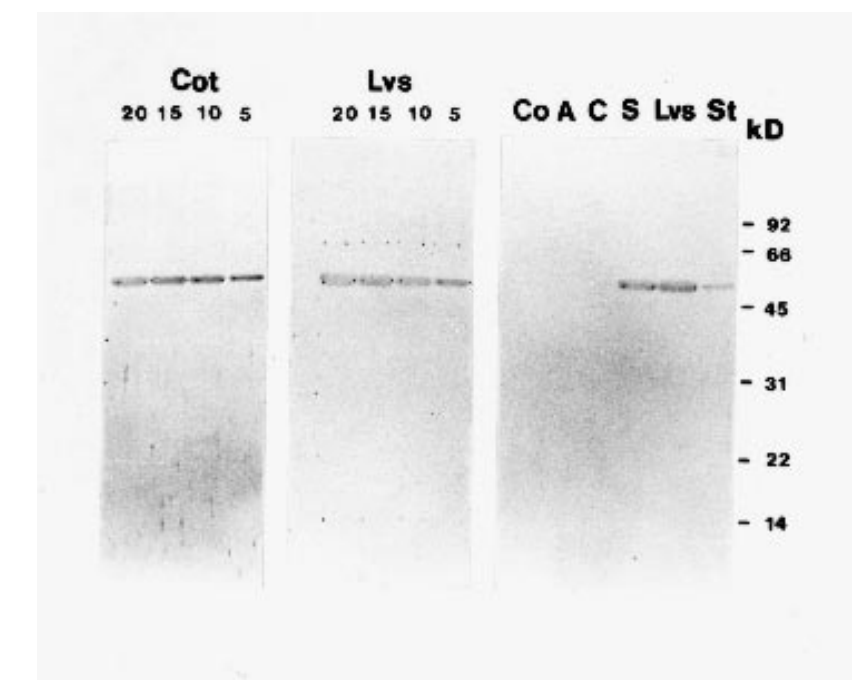

Fig. 2. Immunoblot identification of the LSU of rubisco from various organs. SDSextracted proteins were separated by SDS-PAGE, transferred to nitrocellulose paper and reacted with antisera against the LSU of rubisco from Euglena as described in Materials and Methods. (right) Blot of $20 \mu \mathrm{g}$ protein extracted from corollas (Co), androecia (A), carpels (C), sepals (S), leaves (Lvs) and stems (St); molecular mass markers in kilodaltons are indicated on the right. (middle) Blot of increasing amounts of leaf extract. (left) Blot of increasing amounts of cotyledon extract. Numbers above lanes refer to the amount of SDS-extractable protein loaded.

examined in 2-D gels, significant accumulation of the LSU was also observed as the cotyledons became photosynthetically competent (Bassett et al., 1991).

To quantify the LSU further, SDS-extracted proteins were immunoblotted with antiserum directed against the LSU of rubisco, and band widths were determined for the LSU in increasing amounts of protein extract from photosynthetically competent leaves and cotyledons. A linear relationship between LSU band width and protein concentration $\left(r^{2}=0.98\right)$ was obtained over the range of total protein examined (Fig. 2, Cot and Lvs). When equal

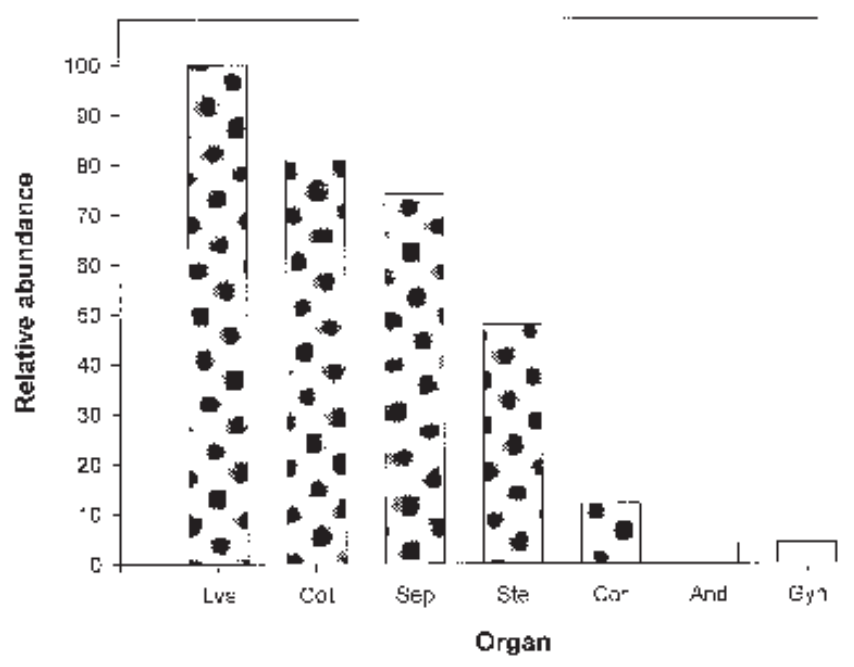

Fig. 3. Relative abundance of rubisco LSU in various organs. Band widths for LSU were measured from $20 \mu \mathrm{g}$ of protein extract separated in gels and immunoblotted to filters. Measurements from $20 \mu \mathrm{g}$ of leaf extract were taken as 100 , and all other organs computed as a percentage. Since the lower limit of detection was estimated to be $5 \%$ of leaf, androecia (And) and gynoecia (Gyn) are graphed at a value of $4 \%$ representing the maximum level of LSU they could express without detection. The data represent an average of two separate experiments. Lvs = leaves; Cot = cotyledons; Sep = sepals; Ste $=$ stems; Cor $=$ corollas. amounts of total protein from the various organs were compared (Fig. 2, right panel), LSU was most abundant in leaves, sepals and stems compared to the inner whorls of the flower. Based on the linear relationship of LSU band width to increasing protein concentration, the relative amounts of LSU were computed for each organ and expressed as percent of LSU in leaf (Fig. 3). These results confirmed relative differences seen in the NEPHGE profiles and indicated that significant levels of LSU were present in both cotyledons (vegetative/storage organ) and sepals (floral). Even mature stems contained nearly $50 \%$ of leaf levels of LSU, whereas the innermost, mature floral whorls expressed $12 \%$ or less compared to leaves.

In a recent study, correlation of rubisco activity and concentration with $\mathrm{CO}_{2}$ assimilation was determined in transgenic tobacco (Nicotiana tabacum L.) containing antisense rubisco small subunit (Hudson et al., 1992). Rubisco activity in 10 independent transformants averaged $18 \%$ of control values, while the average amount of rubisco was estimated at $30 \%$ of the control. This reduction in rubisco level and activity in the transgenic plants resulted in $\mathrm{CO}_{2}$ assimilation rate for the transgenic plants that was $37 \%$ of control values. Furthermore, $\mathrm{CO}_{2}$ assimilation measured in the transgenic plants at high irradiance over a wide range of $\mathrm{CO}_{2}$ partial pressures was essentially linear, indicating that rubisco, not ribulose 1,5 bisphosphate concentration, was rate limiting for $\mathrm{CO}_{2}$ fixation. Examination of the range of rubisco activities seen in individual control plants compared to their $\mathrm{CO}_{2}$ assimilation rates, suggests that rubisco activities as low as $70 \%$ of maximum are sufficient for optimal rates of $\mathrm{CO}_{2}$ assimilation (Hudson et al., 1992).

Rubisco levels in the corollas and reproductive structures of morning glory measured as a function of the LSU were similar to activity measurements in citrus flower buds (Vu et al., 1985), and lower than the average transgenic tobacco rubisco activity cited above. Such a large reduction in LSU (12\% or less than leaf) indicates that mature flower parts are severely limited in their capacity for photosynthesis. While rubisco levels in stems approached $50 \%$ of those in leaves, it is doubtful as to whether these levels are sufficient to maintain significant rates of $\mathrm{CO}_{2}$ assimilation. In contrast, LSU levels in sepals were $74 \%$ of those in mature leaves, raising the possibility that these more leaf-like organs may indeed function photosynthetically like leaves. These levels are close to those of mature cotyledons ( $81 \%$ of leaf) which have been reported capable of photosynthesis in morning glory (Marushige and Marushige, 1966). At least with regard to sepals, levels of rubisco may not be rate limiting for photosynthesis, and further experiments to examine net photosynthesis in sepals must be conducted to determine if they can contribute photosynthetically to developing flowers.

\section{Literature Cited}

Bassett, C.L., C.P. Mothershed, and G.A. Galau. 1988. Floral-specific polypeptides of the Japanese morning glory. Planta 175:221-228.

Bassett, C.L., C.P. Mothershed, and G.A. Galau. 1991. Polypeptide profiles from cotyledons of developing and photoperiodically induced seedlings of the Japanese morning glory (Pharbitis [Ipomoea] nil). J. Plant Growth Regul. 10:147-155.

Bazzaz, F.A., R.W. Carlson, and J.L. Harper. 1979. Contribution to reproductive effort by photosynthesis of flowers and fruits. Nature 279:554-555.

Blake, M.S., K.H. Johnston, G.J. Russel-Jones, and E.C. Gotschlich. 1984. A rapid, sensitive method for detection of alkaline phosphataseconjugated anti-antibody on western blots. Anal. Biochem. 136:175179.

Blanke, M.M. and F. Lenz. 1989. Fruit photosynthesis. Plant Cell Environ. 12:31-46. 
Bravdo, B.A., A. Palgi, S. Lurie, and C. Frenkel. 1977. Changing ribulose diphosphate carboxylase/oxygenase activity in ripening tomato fruit. Plant Physiol. 60:309-312.

Cameron, R.G. and C.L. Bassett. 1988. Inheritance of $\mathrm{C}_{4}$ enzymes associated with carbon fixation in Flaveria species. Plant Physiol. 88:532-536.

Flinn, A.M., C.A. Atkins, and J.S. Pate. 1977. Significance of photosynthetic and respiratory exchanges in the carbon economy of the developing pea fruit. Plant Physiol. 60:412-418.

Gray, J.C. and R.G.O. Kekwick. 1974. An immunological investigation of the structure and function of ribulose 1,5-bisphosphate carboxylase. Eur. J. Biochem. 44:481-489.

Hedley, C.L., D.M. Harvey, and R.J. Keely. 1975. Role of PEP carboxylase during seed development in Pisum sativum. Nature 258:352-354.

Ho, L.C. 1984. Partitioning of assimilates in fruiting tomato plants. Plant Growth Regul. 2:277-285.

Ho, L.C. 1988. Metabolism and compartmentation of imported sugars in sink organs in relation to sink strength. Ann. Rev. Plant Physiol. Plant Mol. Biol. 39:355-378.

Hudson, G.S., J.R. Evans, S. Von Caemmerer, Y.B.C. Arvidsson, and T.J. Andrews. 1992. Reduction of ribulose-1,5-bisphosphate carboxylase/ oxygenase content by antisense RNA reduces photosynthesis in transgenic tobacco plants. Plant Physiol. 98:294-302.

Johal, S. And R. Chollet. 1983. Analysis of catalytic subunit microheterogeneity in ribulosebisphosphate carboxylase/oxygenase from Nicotiana tabacum. Arch. Biochem. Biophys. 223:40-50.

Laemmli, U.K. 1980. Cleavage of structural proteins during the assembly of the head of the bacteriophage T4. Nature 227:680-685.

Laisk, A. and V.M. Oya. 1974. Photosynthesis of leaves subjected to brief pulses of $\mathrm{CO}_{2}$. Sov. J. Plant Physiol. 21:928-935.

Marushige, K. and Y. Marushige. 1966. Effects of light on the appearance of photoperiodic sensitivity of etiolated Pharbitis nil seedlings. Bot. Mag. Tokyo 79:397-403.

Oakley, B.R., D.R. Kirsch, and N.R. Morris. 1980. A simplified ultrasensitive silver stain for detecting proteins in polyacrylamide gels. Anal. Biochem. 105:361-363.

O'Farrell, P.H. 1975. High resolution two dimensional electrophoresis of proteins. J. Biol. Chem. 250:4007-4021.

Pavel, E.W. and T.M. DeJong. 1993. Estimating the photosynthetic contribution of developing peach (Prunus persica) fruits to their growth and maintenance carbohydrate requirements. Physiol. Plant. 88:331-338.

Quebedeaux, B. and R. Chollet. 1975. Growth and development of soybean (Glycine max [L.] Merr.) Pods. Plant Physiol. 55:745-748.

Sharma, V. 1980. Hill activity in chloroplasts from red pigmented corolla, bracts and leaves. Photosynthetica 14:79-82

von Caemmerer, S. and D.L. Edmondson. 1986. Relationship between steady state gas exchange, in vivo ribulose bisphosphate carboxylase activity and some carbon reduction cycle intermediates in Raphanus sativus. Austral. J. Plant Physiol. 13:669-688.

Vu, J.C.V., G. Yelenowski, and M.G. Bausher. 1985. Photosynthetic activity in the flower buds of 'Valencia' orange (Citrus sinensis [L.] Osback). Plant Physiol. 78:420-423.

Weiss, D., M. Schonfeld, and A.H. Halevy. 1989. Photosynthetically active chloroplasts in Petunia corollas. Techniques and New Dev. Photosyn. Res.: NATO Adv. Study Inst. Ser. 168:487-492.

Woodrow, I.E. and J.A. Berry. 1988. Enzymatic regulation of photosynthetic $\mathrm{CO}_{2}$ fixation in $\mathrm{C}_{3}$ plants. Annu. Rev. Plant Physiol. Plant Mol. Biol. 39:533-594. 